\title{
Lyapunov control of bilinear Schrödinger equations
}

\author{
Mazyar Mirrahimi $^{\text {a }}$, Pierre Rouchon $^{\mathrm{b}}$, Gabriel Turinici ${ }^{\mathrm{c}}$ \\ ${ }^{\text {a }}$ Ecole des Mines de Paris, Centre Automatique et Systèmes, 60 Bd Saint-Michel, 75272 Paris cedex 06, FRANCE \\ ${ }^{\mathrm{b}}$ Corresponding author. Ecole des Mines de Paris, Centre Automatique et Systèmes, 60 Bd Saint-Michel, 75272 Paris cedex \\ 06, FRANCE \\ c INRIA Rocquencourt, Domaine de Voluceau, Rocquencourt B.P. 105, 78153 Le Chesnay Cedex, FRANCE and \\ CERMICS-ENPC, Champs sur Marne, 77455 Marne la Vallée Cedex, France
}

\begin{abstract}
A Lyapunov-based approach for trajectory tracking of the Schrödinger equation is proposed. In the finite dimensional case, convergence is precisely analyzed. Connection between the controllability of the linear tangent approximation around the reference trajectory and asymptotic tracking is studied. When the linear tangent approximation is controllable, such a feedback ensures almost global asymptotic convergence. When the linear tangent system is not controllable, the stability of the closedloop system is not asymptotic. To overcome such lack of convergence, we propose, when the reference trajectory is a pure-state, a modification based on adiabatic invariance. Simulations illustrate the simplicity and also the interest for trajectory generation.
\end{abstract}

Key words: Quantum systems, Stabilization, Control Lyapunov function, Adiabatic invariants, Tracking.

\section{Introduction}

Controllability of a finite dimensional quantum system:

$$
\imath \dot{\Psi}=\left(H_{0}+u(t) H_{1}\right) \Psi
$$

where $H_{0}$ and $H_{1}$ are $n \times n$ Hermitian matrices with coefficients in $\mathbb{C}$, can be studied via the general accessibility criteria proposed in [26] and based on Lie-Brackets. More specific results might be found in e.g. [21] and [27]. In particular, the system is controllable if and only if the Lie algebra generated by the skew-symmetric matrices $H_{0} / \imath$ and $H_{1} / \imath$ is $s u(n)$, the Lie algebra of skewHermitian matrices of dimension $n$. Thus controllability of such systems is well characterized. However, such a characterization does not provide in general a simple and efficient way for control design and trajectory generation.

Optimal control techniques (see, e.g., [23] and [13] and the reference herein) provide a first set of methods. An-

Email addresses: mazyar.mirrahimi@ensmp.fr (Mazyar Mirrahimi), pierre.rouchon@ensmp.fr (Pierre Rouchon), gabriel.turinici@inria.fr (Gabriel Turinici). other set consists in using feedback: see,e.g., [20] for decoupling techniques, or $[28,5,25]$ for Lyapunov-based techniques.

In this paper we propose a Lyapunov-based technique that can be relevant for trajectory tracking and generation. The control Lyapunov function is based on the conservation of probability and thus can be used whatever the dimension of the system is. In the finite dimensional case, we show that controllability of the first variation around the reference trajectory is a necessary condition for asymptotic convergence. The analysis is based on an adaptation to bilinear quantum systems of the general method proposed in [8] (see also [6]). Our design method is close but different from the one proposed in $[28,25]$. The Lyapunov function is different and can be adapted to tracking of any system trajectory. Moreover, we propose here to add a fictitious control $\omega$ (see (2)) to take into account the physically meaningless global phase. Such additional fictitious control simplifies notably the control-design and the convergence analysis.

The paper contains three convergence analysis. They are given in theorem 1 when the reference trajectory is a pure-state, in theorem 3 when the reference trajectory is 
adiabatic and in theorem 6 when the reference trajectory is analytic. In section 2 , we introduce the additional fictitious phase control $\omega$, we present the Lyapunov-based tracking feedback and we discuss three simulations that illustrate theorems 1 and 3. This section is tutorial and technicalities are reduced to a strict minimum. The three remaining sections are more technical and formal: section 3 (resp. 4 and 5) is devoted to theorem 1 (resp. 3 and 6 ). In conclusion, we suggest extensions to the multiinput and infinite-dimensional cases.

Preliminary versions of these results can be found in $[18,17]$. Connected but different results can also be found in [19] where Lyapunov design is developed for the density operator $\rho$ instead of the probability amplitudes $\Psi$, and also in [1] that studies the stabilization around degenerate pure state where the linear tangent system is not controllable. The authors thank Claudio Altafini, Jean-Michel Coron and Laurent Praly for interesting discussions and comments.

\section{Tracking feedback design}

\subsection{Dynamics and global phase}

Consider the $n$-states quantum system $(\hbar=1)$

$$
\imath \frac{d}{d t} \Psi=\left(H_{0}+u(t) H_{1}\right) \Psi
$$

where $H_{0}$ and $H_{1}$ are $n \times n$ Hermitian matrices with coefficients in $\mathbb{C}$. Here $H_{0}$ is a time independent Hamiltonian, corresponding to the free evolution of the system in the absence of any external fields. The external interaction here is taken as a control classical field $u(t) \in \mathbb{R}$ coupled to the system through the time independent Hamiltonian $H_{1}$. The wave function $\Psi=\left(\Psi_{i}\right)_{i=1}^{n}$ is a vector in $\mathbb{C}^{n}$, verifying the conservation of probability:

$$
\sum_{i=1}^{n}\left|\Psi_{i}\right|^{2}=1 .
$$

An important characteristic is that the choice of the global phase is arbitrary: physically, the probability amplitudes $\Psi$ and $e^{\imath \theta(t)} \Psi$ describe the same physical state for any global phase $t \mapsto \theta(t) \in \mathbb{R}$.

The conservation of probability and global phase invariance have important consequences on the geometry of the physical state space: $\Psi$ lives on the unit sphere of $\mathbb{C}^{n}$; two probability amplitudes $\Psi_{1}$ and $\Psi_{2}$ are identified when exists $\theta \in \mathbb{R}$ such that $\Psi_{1}=\exp (\imath \theta) \Psi_{2}$. Thus the geometry of the state space does not coincides with the unit sphere of $\mathbb{C}^{n}$, i.e., $\mathbb{S}^{2 n-1}$.
The usual way to take into account such geometry is to reduced the dynamics on the minimal state space: when $n=2$, the dynamics reduces on $\mathbb{S}^{2}$, the unit sphere of $\mathbb{R}^{3}$ called the Bloch sphere. This corresponds to the geometric representation in terms of a fictitious spin $1 / 2$ (see, e.g.,[3]). For $n>2$ such reduction if less simple and the state-space geometry corresponds to the complex projective space of $\mathbb{C}^{n}, \mathbb{P} \mathbb{C}^{n}$.

In this paper, we propose another way to take into account such non trivial geometry of the physical statespace. Instead of reducing the state dimension, we increase the number of control by one. To $u$ we will add a second control $\omega$ corresponding to the time derivative of the global phase. Thus we consider instead of (1) the following control system

$$
\imath \dot{\Psi}=\left(H_{0}+u H_{1}+\omega\right) \Psi
$$

where $\omega \in \mathbb{R}$ is a new control playing the role of a gauge degree of freedom. We can choose it arbitrarily without changing the physical quantities attached to $\Psi$. With such additional fictitious control $\omega$, we will assume in the sequel that the state space is $\mathbb{S}^{2 n-1}$ and the dynamics given by (2) admits two independent controls $u$ and $\omega$.

Adding controls to take into-account symmetry is not new. It has been already proposed for induction motors by Blaschke $[2,12]$. This point has been re-explained in [14] and is widely used for induction motors (see, e.g., point (2) of the discussion page 141 in [4]).

\subsection{Lyapunov control design}

Take a reference trajectory $t \mapsto\left(\Psi_{r}(t), u_{r}(t), \omega_{r}(t)\right)$, i.e., a smooth solution of (2) (or of (1) with $\omega_{r}=0$ ):

$$
\imath \frac{d}{d t} \Psi_{r}=\left(H_{0}+u_{r} H_{1}+\omega_{r}\right) \Psi_{r}
$$

Take the following time varying function $V(\Psi, t)$ :

$$
V(\Psi, t)=\left\langle\Psi-\Psi_{r} \mid \Psi-\Psi_{r}\right\rangle
$$

where $\langle. \mid$.$\rangle denotes the Hermitian product. V$ is positive for all $t>0$ and all $\Psi \in \mathbb{C}^{n}$ and vanishes when $\Psi=\Psi_{r}$. Simple computations show that $V$ is a control Lyapunov function when $\Psi$ satisfies (2):

$$
\begin{array}{r}
\frac{d}{d t} V=2\left(u-u_{r}\right) \Im\left(\left\langle H_{1} \Psi(t) \mid \Psi_{r}\right\rangle\right) \\
+2\left(\omega-\omega_{r}\right) \Im\left(\left\langle\Psi(t) \mid \Psi_{r}\right\rangle\right)
\end{array}
$$

where $\Im$ denotes the imaginary part. With, e.g.,

$$
\begin{aligned}
& u=u_{r}-a \Im\left(\left\langle H_{1} \Psi(t) \mid \Psi_{r}(t)\right\rangle\right) \\
& \omega=\omega_{r}-b \Im\left(\left\langle\Psi(t) \mid \Psi_{r}(t)\right\rangle\right)
\end{aligned}
$$


( $a>0$ and $b>0$ parameters), we ensure $d V / d t \leq 0$.

Let us detail the important case when the reference trajectory corresponds to an equilibrium: $u_{r}=0, \omega_{r}=-\lambda$ and $\Psi_{r}=\phi$ where $\phi$ is an eigen-vector of $H_{0}$ associated to the eigenvalue $\lambda \in \mathbb{R}\left(H_{0} \phi=\lambda \phi,\|\phi\|=1\right)$.Then (5) becomes a static-state feedback:

$$
\begin{gathered}
u=-a \Im\left(\left\langle H_{1} \Psi \mid \phi\right\rangle\right) \\
\omega=-\lambda-b \Im(\langle\Psi \mid \phi\rangle) .
\end{gathered}
$$

\subsection{Tutorial examples and simulations}
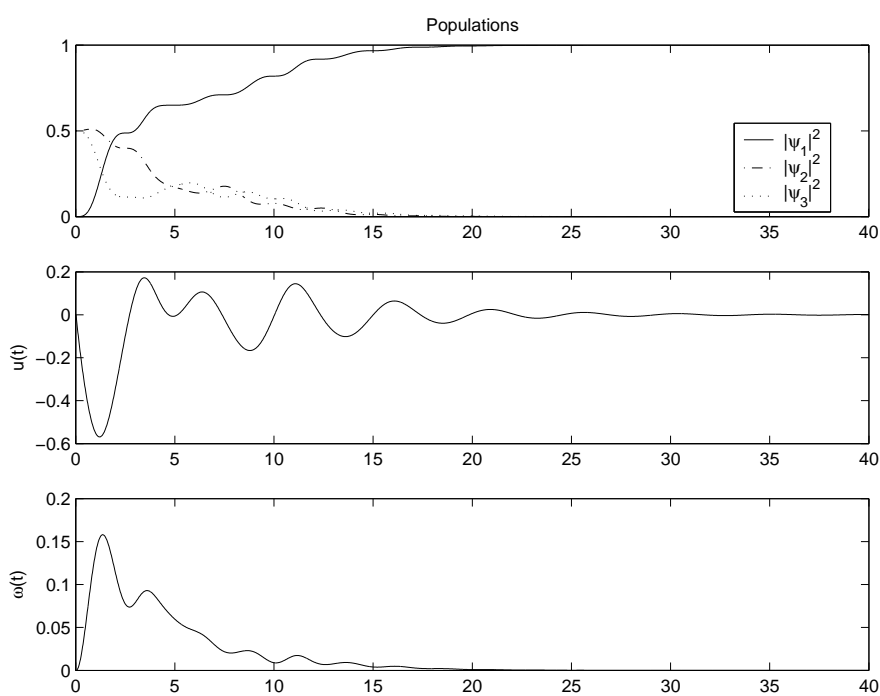

Fig. 1. populations $\left(\left|\Psi_{1}\right|^{2},\left|\Psi_{2}\right|^{2},\left|\Psi_{3}\right|^{2}\right)$ and controls $(u, \omega)$; initial condition $(0,1 / \sqrt{2}, 1 / \sqrt{2})$; system defined by (7) with feedback (8).

Take $n=3, \Psi=\left(\Psi_{1}, \Psi_{2}, \Psi_{3}\right)^{T}$ and

$$
H_{0}=\left(\begin{array}{lll}
0 & 0 & 0 \\
0 & 1 & 0 \\
0 & 0 & \frac{3}{2}
\end{array}\right), \quad H_{1}=\left(\begin{array}{lll}
0 & 1 & 1 \\
1 & 0 & 1 \\
1 & 1 & 0
\end{array}\right)
$$

Let us use the previous Lyapunov control in order to trap our system in the first eigen-state $\phi=(1,0,0)$ of energy $\lambda=0$. We have ( ${ }^{\star}$ means complex conjugate)

$$
\Im\left(\left\langle H_{1} \Psi \mid \phi\right\rangle\right)=\Im\left(\Psi_{2}^{\star}+\Psi_{3}^{\star}\right)
$$

and we take (6) with $a=b=1 / 2$ :

$$
\begin{aligned}
& u=-\frac{1}{2} \Im\left(\Psi_{2}^{\star}+\Psi_{3}^{\star}\right) \\
& \omega=-\frac{1}{2} \Im\left(\Psi_{1}^{\star}\right)
\end{aligned}
$$

Simulations of figure 1 describes the trajectory with $\Psi^{0}=(0,1 / \sqrt{2}, 1 / \sqrt{2})$ as initial state. Other simulations indicate that the trajectories always converge to $\phi$. It appears that such Lyapunov based techniques is quite efficient for system (7). In theorem 1 , it is shown that almost global convergence is equivalent to the controllability of the linear tangent system around $\phi$.
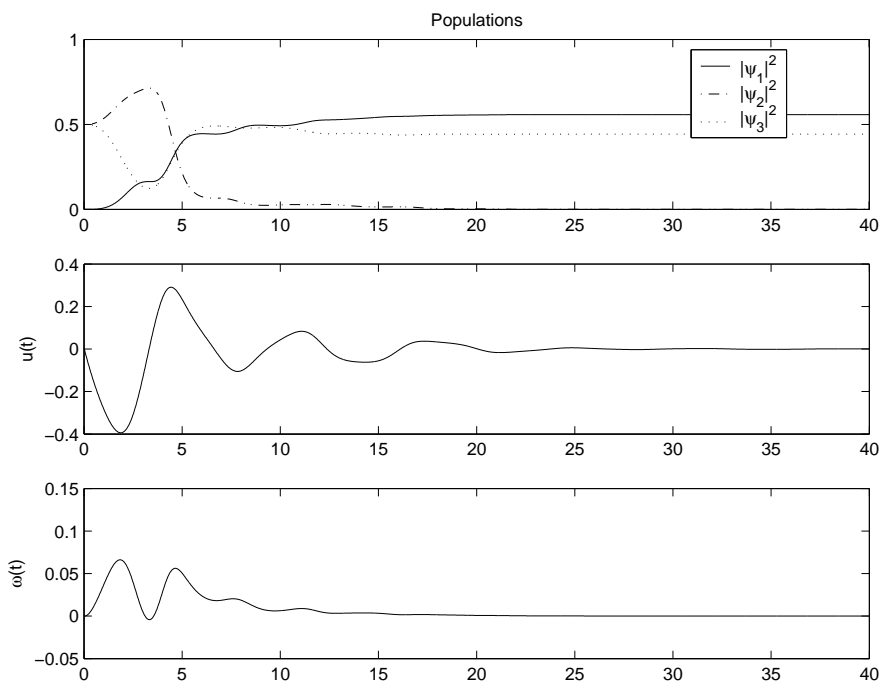

Fig. 2. populations $\left(\left|\Psi_{1}\right|^{2},\left|\Psi_{2}\right|^{2},\left|\Psi_{3}\right|^{2}\right)$ and controls $(u, \omega)$; initial condition $(0,1 / \sqrt{2}, 1 / \sqrt{2}) ; H_{0}$ defined by $(7), H_{1}$ by (9) and feedback by (10).

Let us consider another example that clearly illustrates the limitation of such Lyapunov based technique: $H_{0}$ and the goal state $\phi$ remain unchanged but $H_{1}$ becomes:

$$
H_{1}=\left(\begin{array}{lll}
0 & 1 & 0 \\
1 & 0 & 1 \\
0 & 1 & 0
\end{array}\right)
$$

The feedback becomes

$$
u=-\frac{1}{2} \Im\left(\Psi_{2}^{\star}\right), \quad \omega=-\frac{1}{2} \Im\left(\Psi_{1}^{\star}\right)
$$

Simulations of figure 2 start with $(0,1 / \sqrt{2}, 1 / \sqrt{2})$ as initial condition for $\Psi$. We clearly realize that such a feedback reduces the distance to the first state but does not ensure its convergence to 0 . This is not due to a lack of controllability. This system is controllable since the Lie algebra spanned by $H_{0} / \imath$ and $H_{1} / \imath$ coincides with $s u(3)$ [21]. As explained in theorem 1, such convergence deficiency comes form the fact that the linear tangent system around $\phi$ is not controllable.

To overcome such lack of convergence observed with simulations on figure 2, we will use (5) with a an adiabatic 
reference trajectory

$$
\imath \frac{d}{d t} \Psi_{r}=\left(H_{0}+u_{r}(t) H_{1}\right) \Psi_{r}, \quad \Psi_{r}(0)=(1,0,0),
$$

where $u_{r}=\frac{1}{2} \sin (2 \pi t / T)$ with a period $T=300$, large compared with the natural periods of $H_{0}$ to ensure that $u_{r}$ is a slowly varying time function. Take the following tracking feedback

$$
u=u_{r}-\frac{1}{2} \Im\left(\left\langle H_{1} \Psi \mid \Psi_{r}(t)\right\rangle\right), \quad \omega=-\frac{1}{2} \Im\left(\left\langle\Psi \mid \Psi_{r}(t)\right\rangle\right) .
$$

Since $u_{r}$ varies slowly, adiabatic theory ensures that $\Psi_{r}$ will follow closely the first eigen-state of $H_{0}+u_{r} H_{1}$ [16]. So when $u_{r}$ returns to $0, \Psi_{r}$ will almost return to the first eigen-space spanned by $(1,0,0)$ : we have $\Psi_{r}(T) \approx$ $(\exp (\imath \theta), 0,0)$ for some phase shift $\theta$. If during this slow motion, the reference trajectory $\Psi_{r}$ is in the neighborhood of an eigen-state of $H_{0}+u_{r} H_{1}$ where the linear tangent system is controllable, this will strongly improve convergence. This is effectively the case as shown in figure 3 that illustrates the efficiency of combining Lyapunov design and adiabatic invariance. Detailed results and rigorous proofs of such convergence improvements are given by theorem 6 . See also [1] for a different method based on an implicitly defined control-Lyapunov function that ensures local convergence then the lineartangent system around $\phi$ is not controllable.

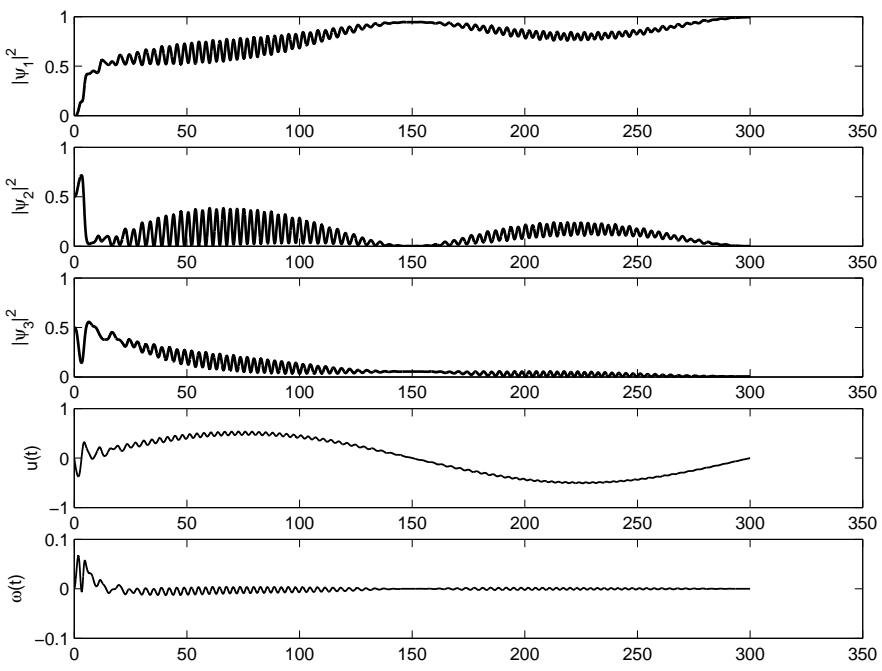

Fig. 3. system and initial conditions identical to figure 2; adiabatic trajectory (11) tracking via the feedback (12).

\section{Convergence analysis}

The goal of this section is to prove the following theorem that underlies simulations of figures 1 and 2 .

Theorem 1 Consider (2) with $\Psi \in \mathbb{S}^{2 n-1}$ and an eigenstate $\phi \in \mathbb{S}^{2 n-1}$ of $H_{0}$ associated to the eigenvalue $\lambda$.
Take the static feedback (6) with $a, b>0$. Then the two following propositions are true:

(1) If the spectrum of $H_{0}$ is not degenerate (all eigenvalues are distinct), the $\Omega$-limit set of the closed loop system is the intersection of $\mathbb{S}^{2 n-1}$ with the real vector-space $E$ spanned by the eigen-vectors $\Phi$ of $H_{0}$ such that $\left\langle H_{1} \Phi \mid \phi\right\rangle=0$ and $\Im(\langle\Phi \mid \phi\rangle)=0$.

(2) The $\Omega$-limit set reduces to $\{\phi,-\phi\}$ if and only if $H_{0}$ is not degenerate and $E=\mathbb{R} \phi$. In this case: the equilibrium $\phi$ is exponentially stable (on $\mathbb{S}^{2 n-1}$ ); the equilibrium $-\phi$ is unstable; the attractor set of $\phi$ is exactly $\mathbb{S}^{2 n-1} /\{-\phi\}$. This case corresponds to the controllability of the linear-tangent system at $\phi$, a time-invariant linear system that lives on the $2 n-1$ plane tangent to $\mathbb{S}^{2 n-1}$ at $\phi$.

For example of figure 1 , it becomes clear that $E=\mathbb{R} \phi$ since $H_{0}$ is not degenerate and $\phi=(1,0,0)$ is almost globally asymptotically stable. Notice the condition $E=$ $\mathbb{R} \phi$ says that, physically, the goal-state $\phi$ is connected to all other pure states via mono-photonic transitions (see, e.g., [16]).

For example of figure 2, elements of $E$ are of the form $(x, 0, z)$ where $x \in \mathbb{R}$ and $z \in \mathbb{C}$; we observe effectively that the $\Omega$-limit set contains elements of the form $(x, 0, r \exp (\imath \theta))$ with $x, r$ and $\theta$ in $\mathbb{R}$ such that $x^{2}+r^{2}=1$. Physically, the transition between $\phi$ and state of energy $3 / 2$ necessitates at least two photons: the feedback (10) cannot find such multi-photonic processes.

The proof of theorem 1 mainly relies on the characterization of the $\Omega$-limit set via LaSalle invariance principle. It provides here a complete description of the invariant subset via the linear system $M \Xi=0$ where $M$ is defined by (14) here below. Such description becomes very simple when $H_{0}$ is not degenerate.

\subsection{Proof of proposition (1) of theorem 1}

Up to a shift on $\omega$ and $H_{0}$, we can assume that $\lambda=$ 0. LaSalle's principle (see, e.g., theorem 3.4, page 115 of [11]) says that the trajectories of the closed-loop system converge to the largest invariant set contained in $d V / d t=0$ where $V$ is defined by (3). The equation $d V / d t=0$ means that

$$
\Im\left(\left\langle H_{1} \Psi \mid \phi\right\rangle\right)=\Im(\langle\Psi \mid \phi\rangle)=0,
$$

Thus $u=0$ and $\omega=0$. Invariance means that $\imath \frac{d}{d t} \Psi=$ $H_{0} \Psi, \frac{d}{d t} \Im\left(\left\langle H_{1} \Psi \mid \phi\right\rangle\right)=0$ and $\frac{d}{d t} \Im(\langle\Psi \mid \phi\rangle)=0$. Clearly $\frac{d}{d t} \Im(\langle\Psi \mid \phi\rangle)=0$ does not give any additional information since $H_{0} \phi=0$. Only $\frac{d}{d t} \Im\left(\left\langle H_{1} \Psi \mid \phi\right\rangle\right)=0$ provides a new independent equation: $\Re\left(\left\langle H_{1} H_{0} \Psi \mid \phi\right\rangle\right)=0$ that reads

$$
\Re\left(\left\langle\left[H_{0}, H_{1}\right] \Psi \mid \phi\right\rangle\right)=0 .
$$


Similarly $\frac{d}{d t} \Re\left(\left\langle\left[H_{0}, H_{1}\right] \Psi \mid \phi\right\rangle\right)=0$ implies

$$
\Im\left(\left\langle\left[H_{0},\left[H_{0}, H_{1}\right]\right] \Psi \mid \phi\right\rangle\right)=0 .
$$

And so on. Finally, the largest invariant set is characterized by $\Im(\langle\Psi \mid \phi\rangle)=0$ with the following conditions,

$$
\begin{aligned}
& \Im\left(\left\langle H_{1} \Psi \mid \phi\right\rangle\right)=0 \\
& \Re\left(\left\langle\left[H_{0}, H_{1}\right] \Psi \mid \phi\right\rangle\right)=0 \\
& \Im\left(\left\langle\left[H_{0},\left[H_{0}, H_{1}\right]\right] \Psi \mid \phi\right\rangle\right)=0
\end{aligned}
$$$$
\text { ... }
$$

that corresponds to the "ad-conditions" obtained in [8]. At each step, we have the Lie bracket of the Hamiltonian $H_{0}$ with the Hamiltonian of the last step.

We can always assume that $H_{0}$ is diagonal. Then we can easily compute the commutator $\left[H_{0}, B\right]$ where $B=\left(B_{i j}\right)$ is a $n \times n$ matrix. With $H_{0}=\operatorname{diag}\left(\lambda_{1}, \ldots, \lambda_{n}\right)$, we have

$$
\left[H_{0}, A\right]_{i, j}=\left(\lambda_{i}-\lambda_{j}\right) B_{i j}
$$

Let take $B=H_{1}$ in order to simplify the notations. So:

$$
\begin{aligned}
{\left[H_{0}, B\right] } & =\left(\left(\lambda_{i}-\lambda_{j}\right) B_{i j}\right) \\
{\left[H_{0},\left[H_{0}, B\right]\right] } & =\left(\left(\lambda_{i}-\lambda_{j}\right)^{2} B_{i j}\right) \\
\vdots & \\
\underbrace{\left[H_{0},\left[H_{0}, \ldots,\left[H_{0}\right.\right.\right.}_{k \text { times }}, B]] \ldots] & =\left(\left(\lambda_{i}-\lambda_{j}\right)^{k} B_{i j}\right) .
\end{aligned}
$$

Thus the previous system reads:

$$
\begin{aligned}
& \Im\left(\Sigma_{j} B_{1 j} \Psi_{j}\right)=0, \\
& \Re\left(\Sigma_{j}\left(\lambda_{1}-\lambda_{j}\right) B_{1 j} \Psi_{j}\right)=0, \\
& \vdots \\
& \Im\left(\Sigma_{j}\left(\lambda_{1}-\lambda_{j}\right)^{2 k} B_{1 j} \Psi_{j}\right)=0, \\
& \Re\left(\Sigma_{j}\left(\lambda_{1}-\lambda_{j}\right)^{2 k+1} B_{1 j} \Psi_{j}\right)=0, \\
& \ldots
\end{aligned}
$$

Set

$$
M=\left(\begin{array}{ccc}
1 & \ldots & 1 \\
\left(\lambda_{1}-\lambda_{2}\right)^{2} & \ldots & \left(\lambda_{1}-\lambda_{n}\right)^{2} \\
\left(\lambda_{1}-\lambda_{2}\right)^{4} & \ldots & \left(\lambda_{1}-\lambda_{n}\right)^{4} \\
\vdots & \vdots & \vdots \\
\left(\lambda_{1}-\lambda_{2}\right)^{2(n-2)} & \ldots & \left(\lambda_{1}-\lambda_{n}\right)^{2(n-2)}
\end{array}\right)
$$

and

$$
\Xi=\left(\left(1+\imath\left(\lambda_{1}-\lambda_{2}\right)\right) B_{12} \Psi_{2}, \ldots,\left(1+\imath\left(\lambda_{1}-\lambda_{n}\right)\right) B_{1 n} \Psi_{n}\right)^{T}
$$

Then (13) implies that $M \Xi=0$. Indeed even if (13) corresponds to an infinite number of linear relations, using the Vandermonde structure of these equations one can easily see that it suffices to consider its first $n-1$ equations: higher order equations are necessarily linear combinations of the first $n-1$ equations. Thus the linear system $M \Xi=0$ with $\Im(\langle\Psi \mid \phi\rangle)=0$ provides a complete characterization of the $\Omega$-limit set.

In the particular case where the free Hamiltonian $H_{0}$ has a non-degenerate spectrum, $M$ is invertible and thus $\Xi=0$. Then $\Psi \in \mathbb{S}^{2 n-1}$ is in the $\Omega$-limit set if and only if

$$
B_{1 j} \Psi_{j}=0, \forall j \in\{2, \ldots, n\}
$$

and $\Im\left(\Psi_{1}\right)=0$.

\subsection{Proof of proposition (2) of theorem 1}

Notice first that in any case the $\Omega$-limit set contains $\phi$ and $-\phi$.

If $H_{0}$ has a non-degenerate spectrum and $E=\mathbb{R} \phi$ then proposition (1) implies that the $\Omega$-limit set is just $\{ \pm \phi\}$. Now let us suppose that at least one of these two conditions is not fulfilled.

Assume that $E \neq \mathbb{R} \phi$. Thus exists an eigen-vector $\Phi$ of $H_{0}$ not co-linear to $\phi$ such that $\left\langle H_{1} \Phi \mid \phi\right\rangle=0$. With $\Psi(0)=\Phi$ as initial state, we have $u(t)=0$ and $\omega(t)=$ $-\lambda$ and $\Psi(t)=\Phi$ for all $t>0$. The $\Omega$-limit set contains $\Phi$.

Assume $E=\mathbb{R} \phi$ but that $H_{0}$ has a degenerate spectrum. We will consider two cases:

(1) There exists an eigen-vector $\phi_{k}$ with length 1 of $H_{0}$ orthogonal to $\phi$ but with the same eigen-value $\lambda$. Since $\left.E=\mathbb{R} \phi, B_{1 k}=\left\langle H_{1} \phi_{k} \mid \phi\right\rangle\right) \neq 0$. With $\Psi(0)=\frac{B_{1 k}}{\left|B_{1 k}\right|} \phi_{k}$ as initial state, we have $u(t)=0$, $\omega=-\lambda$ and $\Psi(t)=\frac{B_{1 k}}{\left|B_{1 k}\right|} \phi_{k}$ belongs to the $\Omega$-limit set.

(2) There exist two orthogonal eigen-vectors $\phi_{k}$ and $\phi_{l}$ of $H_{0}$, with length one and admitting the eigenvalues $\mu \neq \lambda$. Since $\left.E=\mathbb{R} \phi, B_{1 k}=\left\langle H_{1} \phi_{k} \mid \phi\right\rangle\right) \neq 0$ and $\left.B_{1 l}=\left\langle H_{1} \phi_{l} \mid \phi\right\rangle\right) \neq 0$. With $\Psi(0)=\left(B_{1 k} \phi_{l}-\right.$ $\left.B_{1 l} \phi_{k}\right) / \sqrt{\left|B_{1 k}\right|^{2}+\left|B_{1 l}\right|^{2}}$, we have $u(t)=0, \omega=$ $-\lambda$ and

$$
\Psi(t)=e^{-\imath(\mu-\lambda) t} \Psi(0) .
$$

Thus the $\Omega$-limit set contains $\left(e^{\imath \alpha} \Psi(0)\right)_{\alpha \in[0,2 \pi]}$.

The proof of the first part of proposition (2) is thus done.

Let us prove now that $\phi$ is locally exponentially stable when $H_{0}$ is not degenerate and $E=\mathbb{R} \phi$. We will prove 
that the linear tangent closed-loop system is asymptotically stable. This will automatically implies that the equilibrium $\phi$ is locally exponentially stable. Set

$$
\Psi(t)=\phi+\Delta \Psi(t)
$$

with $\Delta \Psi$ small. Then up to second order terms we have

$$
\begin{gathered}
\imath \frac{d}{d t} \Delta \Psi=\left(H_{0}-\lambda I\right) \Delta \Psi-a \Im\left(\left\langle H_{1} \Delta \Psi \mid \phi\right\rangle\right) H_{1} \phi \\
-b \Im(\langle\Delta \Psi \mid \phi\rangle) \phi
\end{gathered}
$$

and $\Re(\langle\Delta \Psi \mid \phi\rangle)=0$ (definition of the tangent space at $\phi$ to the unit sphere $\left.\mathbb{S}^{2 n-1}\right)$. Set $W(\Delta \Psi)=\frac{1}{2}\langle\Delta \Psi \mid \Delta \Psi\rangle$. Simple computations show that $d W / d t \leq 0$ and $E=\mathbb{R} \phi$ implies that the LaSalle's invariant set of this linearized system reduces to $\Delta \Psi=0$ on the tangent space at $\phi$ to $\mathbb{S}^{2 n-1}$.

The fact that $-\phi$ is unstable results from the fact that the Lyapunov function $V$ reaches its maximum on $\mathbb{S}^{2 n-1}$ only for $\Psi=-\phi$. Thus if $\Psi(0) \neq-\phi$, then necessary $\Psi(t)$ must converge to the other point of the $\Omega$-limit set. Thus $\lim _{t \mapsto+\infty} \Psi(t)=\phi$; the equilibrium $-\phi$ is unstable, the attraction region of $\phi$ is $\mathbb{S}^{2 n-1} /\{-\phi\}$.

Let us finally prove that $H_{0}$ non degenerate and $E=\mathbb{R} \phi$ is equivalent to the controllability of the linear-tangent system at $\phi$.

Set $\Psi(t)=\phi+\Delta \Psi(t)$ with $\Re(\langle\Delta \Psi \mid \phi\rangle)=0, u=\Delta u$ and $\omega=-\lambda+\Delta \omega$ with $\Delta \Psi, \Delta u$ and $\Delta \omega$ small. Then up to second order terms, $(2)$ reads

$$
\imath \frac{d}{d t} \Delta \Psi=\left(H_{0}-\lambda I\right) \Delta \Psi+\Delta u H_{1} \phi+\Delta \omega \phi
$$

Take $\left(\phi_{1}, \ldots, \phi_{n}\right)$ an ortho-normal eigen-basis of $H_{0}$ associated to $\left(\lambda_{1}, \ldots, \lambda_{n}\right)$ with $\phi_{1}=\phi$ and $\lambda_{1}=\lambda$. Set $\left(z_{1}, \ldots, z_{n}\right) \in \mathbb{C}^{n}$ the coordinates of $\Delta \Psi$ in this basis. Then: $\Re\left(z_{1}\right)=0$ and

$$
\begin{aligned}
\frac{d}{d t}\left(\Im\left(z_{1}\right)\right) & =-\Delta \omega-B_{11} \Delta u \\
\imath \frac{d}{d t} z_{2} & =\left(\lambda_{2}-\lambda_{1}\right) z_{2}+B_{12} \Delta u \\
& \vdots \\
\imath \frac{d}{d t} z_{n} & =\left(\lambda_{n}-\lambda_{1}\right) z_{n}+B_{1 n} \Delta u
\end{aligned}
$$

where $B_{i j}=\left\langle\phi_{i} \mid H_{1} \phi_{j}\right\rangle$. Controllability is then equivalent to the fact that $B_{1 i} \neq 0$ and $\lambda_{i} \neq \lambda_{j}$ for $i \neq j$ (use, e.g., Kalman controllability matrix). This is clearly equivalent to $H_{0}$ non degenerate and $E=\mathbb{R} \phi$.

\subsection{A technical lemma}

The following lemma will be used during the proof of theorem 3 .

Lemma 2 Consider (2). Take $\phi \in \mathbb{S}^{2 n-1}$ an eigenvector of $H_{0}$ associated to the eigen-value $\lambda$. Assume that $H_{0}$ is not degenerate and the vector-space $E$ defined in theorem 1 coincides with $\mathbb{R} \phi$. Take $\theta \in \mathbb{R}$ and consider the following closed-loop system $(a, b>0)$

$$
(\Upsilon)\left\{\begin{aligned}
\imath \frac{d}{d t} \Psi & =\left(H_{0}+u H_{1}+\omega\right) \Psi \\
u & =-a \Im\left(\left\langle H_{1} \Psi \mid e^{\imath(\theta-\lambda t)} \phi\right\rangle\right) \\
\omega & =-b \Im\left(\left\langle\Psi \mid e^{\imath(\theta-\lambda t)} \phi\right\rangle\right)
\end{aligned}\right.
$$

Then for all $\eta>0$ and $\epsilon>0$, exists $T>0$, such that for all $\theta \in \mathbb{R}$ and $\Psi^{0} \in \mathbb{C}^{n}$ satisfying $\left\|\Psi^{0}-\exp (\imath \theta) \phi\right\| \leq 2-\eta$, we have

$$
\forall t \geq T, \quad \min _{\alpha \in[0,2 \pi]}\|\Psi(t)-\exp (\imath \alpha) \phi\| \leq \epsilon
$$

where $\Psi$ is the solution of $(\Upsilon)$ with $\Psi(0)=\Psi^{0}$.

Notice that $T$ is independent of $\theta$ : this point will be crucial in the proof of theorem 3. The detailed proof of this lemma is left to the reader. It relies on the following arguments:

- Up to a shift of $-\lambda$ on $\omega$ and $H_{0}$, and multiplying $\Psi$ by $e^{-\imath \theta}$, we can assume $\lambda=0$ and $\theta=0$; one recognizes feedback (6).

- $\|\Psi-\phi\|$ is a Lyapunov function that reaches its maximum value 2 only for $\Psi=-\phi$.

- $\Psi$ lives on the compact $\mathbb{S}^{2 n-1}$ and according to proposition (2) of theorem 1 , the $\Omega$-limit set of $(\Upsilon)$ is made of two equilibrium $\{\phi,-\phi\}$ with $\phi$ exponentially stable with attraction region $\mathbb{S}^{2 n-1} /\{-\phi\}$.

- The time taken by $\Psi(t)$ to enter the sphere of center $\phi$ and radius $\epsilon$ is a continuous function of $\Psi(0) \epsilon$ $\mathbb{S}^{2 n-1} /\{-\phi\}$. It reaches its maximum on every compact subset of $\mathbb{S}^{2 n-1} /\{-\phi\}$.

\section{Lyapunov tracking of adiabatic trajectories}

The goal of this section is to prove the following theorem that underlies simulations of figure 3 .

Theorem 3 Consider (2) and an analytic map $u \mapsto$ $\left(\phi^{u}, \lambda^{u}\right)$ where $\phi^{u}$ is an eigenvector of $H_{0}+u_{1} H_{1}$ of length 1 associated to the eigenvalue $\lambda^{u}$. Take a smooth map $f:[0,1] \mapsto[0,1]$ such that $f(0)=f(1)=0$. For $T>0$, denote by $[0, T] \ni t \mapsto \Psi_{r}(t)$ the reference trajectory 
solution of

$$
\left(\Sigma_{r}\right)\left\{\begin{aligned}
\imath \frac{d}{d t} \Psi_{r} & =\left(H_{0}+u_{r}(t) H_{1}\right) \Psi_{r} \\
\Psi_{r}(0) & =\phi^{0} \\
u_{r}(t) & =f(t / T) .
\end{aligned}\right.
$$

and by $[0, T] \ni t \mapsto \Psi(t)$ the trajectory of closed-loop system (see (5), $a, b>0$ constant)

$$
(\Sigma)\left\{\begin{aligned}
\imath \frac{d}{d t} \Psi & =\left(H_{0}+u(t) H_{1}+\omega\right) \Psi \\
\Psi(0) & =\Psi^{0} \\
u & =u_{r}(t)-a \Im\left(\left\langle H_{1} \Psi \mid \Psi_{r}\right\rangle\right) \\
\omega & =-b \Im\left(\left\langle\Psi \mid \Psi_{r}\right\rangle\right) .
\end{aligned}\right.
$$

Assume that exists $\bar{s} \in] 0,1[$ such that the linear tangent system of (2) around the steady state $\bar{\Psi}=\phi^{\bar{u}}$, $\bar{u}=f(\bar{s})=u_{r}(\bar{s} T)$ and $\bar{\omega}=-\lambda^{\bar{u}}$ is controllable.

Then for all $\eta>0$ and $\epsilon>0$, exists $\bar{T}>0$, such that for all $\Psi^{0} \in \mathbb{S}^{2 n-1}$ such that $\left\|\Psi^{0}-\phi^{0}\right\| \leq 2-\eta$ we have

$$
\forall T \geq \bar{T}, \quad \min _{\alpha \in[0,2 \pi]}\left\|\Psi(T)-e^{\imath \alpha} \phi^{0}\right\| \leq \epsilon .
$$

The existence of the analytical map $u \mapsto\left(\phi^{u}, \lambda^{u}\right)$ comes from the following classical result of the perturbation theory for finite dimensional self-adjoint operators ([10] page 121$)$ :

Lemma 4 Let us consider the $n \times n$ hermitian matrices $H_{0}$ and $H_{1}$ with entries in $\mathbb{C}$ and let us define

$$
H(u):=H_{0}+u H_{1} .
$$

For each real $u \in \mathbb{R}$, there exists an orthonormal basis $\left(\phi_{j}^{u}\right)_{j \in\{1, \ldots, n\}}$ of $\mathbb{C}^{n}$ consisting of eigenvectors of $H(u)$. These orthonormal eigenvectors can be chosen as analytic functions of $u \in \mathbb{R}$.

For the case of figure 3 , it is then clear that the eigenvector $\phi^{0}=(1,0,0)$ of $H_{0}$ belongs to such an analytic branch. Moreover simple numerical computations indicate that for $\bar{u}=0.1$, the tangent system around $\phi^{\bar{u}}$ is controllable. Moreover, since $\phi^{0}$ is defined up a multiplication by $e^{\imath \theta}, \theta \in[0,2 \pi]$, one can always choose $\phi^{0}$ such that $\left\|\Psi^{0}-\phi^{0}\right\| \leq 1$. Thus, all the conditions of theorem 3 are fulfilled and we can adjust the final error by taking $T$ large enough. As suggested here below, one observes that, asymptotically when $\epsilon>0$ tends to 0 , the required time $T$ to ensure a final error less than $\epsilon$ increases as $-k \log \epsilon$ for some $k>0$. Such asymptotics for $T$ can be interpreted as a kind of exponential convergence.

The proof of theorem 3 relies on the following adiabatic theorem borrowed from $[7,9]$
Theorem 5 Consider the solution $[0, T] \ni t \mapsto \Psi_{r}(t)$ of $\left(\Sigma_{r}\right)$. Then for all $\epsilon>0$, there exists $T_{\epsilon}>0$ such that for all $T \geq T_{\epsilon}$,

$$
\forall t \in[0, T], \quad \min _{\alpha \in[0,2 \pi]}\left\|\Psi_{r}(t)-e^{\imath \alpha} \phi^{u_{r}(t)}\right\| \leq \epsilon .
$$

In fact, there exist precise results based on exponentially precise estimates: the dependence of $T_{\epsilon}$ is linear in $\log \epsilon$ (see, e.g., theorem(2.1) another version of adiabatic theorems presented in [15]; see also [22]). An enhanced version of theorem 5 can be obtained where the dependence of $\bar{T}$ versus $\epsilon>0$ is linear in $\log \epsilon$ as $\epsilon \rightarrow 0$. The remaining part of this section is devoted to the proof of theorem 3.

PROOF. Take $\eta>0, \epsilon>0$ and $T>0$. Denote by $\mathbb{R} \ni t \mapsto \tilde{\Psi}(t)$ the solution of the following closed-loop system

$(\tilde{\Sigma})$

$$
\left\{\begin{aligned}
\imath \frac{d}{d t} \tilde{\Psi} & =\left(H_{0}+\tilde{u} H_{1}+\tilde{\omega}\right) \tilde{\Psi} \\
\tilde{\Psi}(\bar{s} T) & =\Psi(\bar{s} T) \\
\tilde{u} & =\bar{u}-a \Im\left(\left\langle H_{1} \tilde{\Psi} \mid e^{\imath\left(\bar{\theta}-(t-\bar{s} T) \lambda^{\bar{u}}\right)} \phi^{\bar{u}}\right\rangle\right) \\
\tilde{\omega} & =-b \Im\left(\left\langle\tilde{\Psi} \mid e^{\imath\left(\bar{\theta}-(t-\bar{s} T) \lambda^{\bar{u}}\right)} \phi^{\bar{u}}\right\rangle\right)
\end{aligned}\right.
$$

where the angle $\bar{\theta} \in[0,2 \pi]$ is such that

$$
\left\|\Psi_{r}(\bar{s} T)-e^{\imath \bar{\theta}} \phi^{\bar{u}}\right\|=\min _{\alpha \in[0,2 \pi]}\left\|\Psi_{r}(\bar{s} T)-e^{\imath \alpha} \phi^{\bar{u}}\right\| .
$$

By the adiabatic theorem 5 , exists $T_{a}>0$ such that for all $T \geq T_{a}$ :

$$
\forall t \in[0, T], \min _{\alpha \in[0,2 \pi]}\left\|\Psi_{r}(t)-e^{\imath \alpha} \phi^{u_{r}(t)}\right\| \leq \frac{\eta}{2} .
$$

Since $\left\|\Psi-\Psi_{r}\right\|$ is a time decreasing function we have

$$
\left\|\Psi(\bar{s} T)-\Psi_{r}(\bar{s} T)\right\| \leq\left\|\Psi(0)-\Psi_{r}(0)\right\| \leq 2-\eta
$$

But for $T \geq T_{a},\left\|\Psi_{r}(\bar{s} T)-e^{\imath \bar{\theta}} \phi^{\bar{u}}\right\| \leq \eta / 2$. Thus for $T \geq T_{a}$,

$$
\begin{aligned}
& \left\|\Psi(\bar{s} T)-e^{\imath \bar{\theta}} \phi^{\bar{u}}\right\| \leq \\
& \quad\left\|\Psi(\bar{s} T)-\Psi_{r}(\bar{s} T)\right\|+\left\|\Psi_{r}(\bar{s} T)-e^{\imath \bar{\theta}} \phi^{\bar{u}}\right\| \leq 2-\eta / 2
\end{aligned}
$$

Lemma 2 applied on $(\tilde{\Sigma})$ provides a $T_{b}>0$ such that

$$
\forall t \geq T_{b}, \min _{\alpha \in[0,2 \pi]}\left\|\tilde{\Psi}(\bar{s} T+t)-e^{\imath \alpha} \phi^{\bar{u}}\right\| \leq \frac{\epsilon}{3}
$$

One can always choose $T_{a}$ large enough to ensure that for all $T \geq T_{a}, \bar{s} T+T_{b} \leq T, \bar{s}<1$ and $T_{b}$ is independent 
of $T>T_{a}$. This last point will be crucial in the sequel: it results from the invariance with respect to time translation of lemma 2 and from the independence of $T_{b}$ versus $\bar{\theta}$ that depends a priori on $T$.

Let us compare now the solution of $(\Sigma)$ and $(\tilde{\Sigma})$ for $t \in\left[\bar{s} T, \bar{s} T+T_{b}\right]$. Both systems have for $t=\bar{s} T$ the same initial value. They are both closed-loop dynamics (dynamics (2) with the tracking feedback (5)). The only differences are the reference trajectories : $t \mapsto \Psi_{r}(t)$ for $(\Sigma)$ and $t \mapsto e^{\imath\left(\bar{\theta}-(t-\bar{s} T) \lambda^{\bar{u}}\right)} \phi^{\bar{u}}$ for $(\tilde{\Sigma})$.

Let us prove that

$$
\lim _{T \rightarrow+\infty}\left(\sup _{t \in\left[\bar{s} T, \bar{s} T+T_{b}\right]}\left\|\Psi_{r}(t)-e^{\imath\left(\bar{\theta}-(t-\bar{s} T) \lambda^{\bar{u}}\right)} \phi^{\bar{u}}\right\|\right)=0 .
$$

For $T$ large, these two reference trajectories satisfy on $\left[\bar{s} T, \bar{s} T+T_{b}\right]$ almost the same differential equations (1) with almost the same initial conditions at $t=\bar{s} T$ : $\max _{t \in\left[\bar{s} T, \bar{s} T+T_{b}\right]}\left|u_{r}(t)-\bar{u}\right|$ and $\left|\Psi_{r}(\bar{s} T)-e^{\imath \bar{\theta}} \phi^{\bar{u}}\right|$ tend to 0 as $T$ tends to $+\infty$. Since the interval length $T_{b}$ does not depends on $T$, we have (17) by standard continuity arguments.

For the same reasons, (17) implies for $(\Sigma)$ and $(\tilde{\Sigma})$ :

$$
\lim _{T \rightarrow+\infty}\left(\sup _{t \in\left[\bar{s} T, \bar{s} T+T_{b}\right]}\|\Psi(t)-\tilde{\Psi}(t)\|\right)=0 .
$$

Thus there exists $\bar{T}>T_{a}$ such that for all $T \geq \bar{T}$ we have via (17) and (18):

$$
\begin{aligned}
\left\|\Psi\left(\bar{s} T+T_{b}\right)-\tilde{\Psi}\left(\bar{s} T+T_{b}\right)\right\| & \leq \frac{\epsilon}{3} \\
\left\|\Psi_{r}\left(\bar{s} T+T_{b}\right)-e^{\imath\left(\bar{\theta}-T_{b} \lambda^{\bar{u}}\right)} \phi^{\bar{u}}\right\| & \leq \frac{\epsilon}{3} .
\end{aligned}
$$

Since

$$
\begin{aligned}
& \left\|\Psi\left(\bar{s} T+T_{b}\right)-\Psi_{r}\left(\bar{s} T+T_{b}\right)\right\| \leq \\
& \left\|\Psi\left(\bar{s} T+T_{b}\right)-\tilde{\Psi}\left(\bar{s} T+T_{b}\right)\right\| \\
& +\left\|\tilde{\Psi}\left(\bar{s} T+T_{b}\right)-e^{\imath\left(\bar{\theta}-T_{b} \lambda^{\bar{u}}\right)} \phi^{\bar{u}}\right\| \\
& +\left\|e^{\imath\left(\bar{\theta}-T_{b} \lambda^{\bar{u}}\right)} \phi^{\bar{u}}-\Psi_{r}\left(\bar{s} T+T_{b}\right)\right\|
\end{aligned}
$$

we have, for $T \geq \bar{T}$

$$
\left\|\Psi\left(\bar{s} T+T_{b}\right)-\Psi_{r}\left(\bar{s} T+T_{b}\right)\right\| \leq \epsilon .
$$

Since $\left\|\Psi-\Psi_{r}\right\|$ is a decreasing time function, we conclude that, for $T \geq \bar{T}$,

$$
\left\|\Psi(T)-\Psi_{r}(T)\right\| \leq\left\|\Psi\left(\bar{s} T+T_{b}\right)-\Psi_{r}\left(\bar{s} T+T_{b}\right)\right\| \leq \epsilon
$$

since $\bar{s} T+T_{b} \leq T$.

\section{$5 \quad$ Trajectory tracking}

The goal of this section is to propose a generalization to any analytic reference trajectory $\Psi_{r}$ of proposition (2) in theorem 1. Let us first write the complex dynamics as a real dynamics in $\mathbb{R}^{2 n}$.

With $\Re$ and $\Im$ for real part and imaginary part, set

$$
\begin{gathered}
\widetilde{\Psi}=(\Re(\Psi), \Im(\Psi))^{T}, \quad \widetilde{\Psi}_{r}=\left(\Re\left(\Psi_{r}\right), \Im\left(\Psi_{r}\right)\right)^{T}, \\
G_{0}=\left(\begin{array}{cc}
\Im\left(H_{0}\right) & \Re\left(H_{0}\right) \\
-\Re\left(H_{0}\right) & \Im\left(H_{0}\right)
\end{array}\right), \\
G_{1}=\left(\begin{array}{cc}
\Im\left(H_{1}\right) & \Re\left(H_{1}\right) \\
-\Re\left(H_{1}\right) & \Im\left(H_{1}\right)
\end{array}\right), \\
G_{2}=\left(\begin{array}{cc}
0 & I d \\
-I d & 0
\end{array}\right) .
\end{gathered}
$$

The real matrices $G_{0}, G_{1}$ and $G_{2}$ are skew-symmetric. Now the closed-loop dynamics (2) with (5) reads:

$$
\begin{aligned}
\frac{d}{d t} \widetilde{\Psi} & =\left(G_{0}+u G_{1}+\omega G_{2}\right) \widetilde{\Psi} \\
u & =u_{r}-a\left\langle G_{1} \widetilde{\Psi} \mid \widetilde{\Psi}_{r}\right\rangle_{\mathbb{R}^{2 n}} \\
\omega & =\omega_{r}-b\left\langle G_{2} \widetilde{\Psi} \mid \widetilde{\Psi}_{r}\right\rangle_{\mathbb{R}^{2 n}}
\end{aligned}
$$

with $\langle. \mid \cdot\rangle_{\mathbb{R}^{2 n}}$ the Euclidean product in $\mathbb{R}^{2 n}$. Set

$$
\begin{aligned}
A(t) & =G_{0}+u_{r}(t) G_{1}+\omega_{r}(t) G_{2} \\
b_{1}(t) & =G_{1} \widetilde{\Psi}_{r}(t) \\
b_{2}(t) & =G_{2} \widetilde{\Psi}_{r}(t) \\
B(t) & =\left(b_{1}(t), b_{2}(t)\right)
\end{aligned}
$$

Then the linear tangent system around the reference trajectory $\left(\widetilde{\Psi}_{r}, u_{r}, \omega_{r}\right)$ reads:

$$
\frac{d}{d t} \widetilde{\delta \Psi}=A(t) \widetilde{\delta \Psi}+\delta u b_{1}(t)+\delta \omega b_{2}(t) .
$$

where $\widetilde{\delta \Psi}$ evolves in the tangent space to $\mathbb{S}^{2 n-1}$ at $\widetilde{\Psi}$ : $\widetilde{\delta \Psi}(t)$ is an element of

$$
\widetilde{\mathbf{T}}^{2 n-1}(t)=\left\{v \in \mathbb{R}^{2 n} \mid\left\langle v \mid \widetilde{\Psi}_{r}(t)\right\rangle_{\mathbb{R}^{2 n}}=0\right\} .
$$

As $u_{r}$ and $\omega_{r}$ are analytic functions of $t$, so are $\widetilde{\Psi}_{r}(t)$, $A(t), b_{1}(t)$ and $b_{2}(t)$. Thus, using the generalized Kalman criteria for analytic time-dependent linear systems [24], controllability of (21) is equivalent to:

$\operatorname{span}\left(\operatorname{Range}\left(\left[A(t)-\frac{d}{d t}\right]^{i} B(t)\right) ; i \in \mathbb{N}\right)=\widetilde{\mathbf{T}}^{2 n-1}(t)$. 
Since the dependence in $t$ is analytic, controllability of the tangent system around time $t_{0}$ is equivalent to controllability around any time $t$.

Theorem 6 Consider (19) where

$$
[0,+\infty) \ni t \mapsto\left(\widetilde{\Psi}_{r}(t), u_{r}(t), \omega_{r}\right)
$$

is an analytic reference trajectory. The following two implications are true:

(1) Assume that there exists $\eta>0$ such that

$$
\forall \widetilde{\Psi}(0) \in \mathbb{S}^{2 n-1},\left\|\widetilde{\Psi}(0)-\widetilde{\Psi}_{r}(0)\right\| \leq \eta
$$

we have

$$
\lim _{t \rightarrow+\infty}\left\|\widetilde{\Psi}(t)-\widetilde{\Psi}_{r}(t)\right\|=0 .
$$

Then the linear tangent system (21) is controllable.

(2) Assume that $\forall i \in \mathbb{N}$, $\sup _{t>0}\left|u_{r}^{(i)}(t)\right|<+\infty$ and $\sup _{t \geq 0}\left|\omega_{r}^{(i)}(t)\right|<+\infty$. Assume also that there exists a sequence $\left\{t_{k}\right\}_{k=1}^{\infty}$ with $\lim _{k \rightarrow+\infty} t_{k}=+\infty$ such that $\lim _{k \rightarrow+\infty} \widetilde{\Psi}_{r}\left(t_{k}\right)$ exists and equals $\widetilde{\Psi}_{r}^{\infty} \in$ $\mathbb{S}^{2 n-1}$ and such that $\forall i \in \mathbb{N}$

$$
\left.\lim _{k \rightarrow+\infty}\left(\left[A-\frac{d}{d t}\right]^{i} B\right)\right|_{t=t_{k}}
$$

exists and equals $B_{i}^{\infty}(A$ and $B$ are defined in (20)). Assume finally that the vector space spanned by $\widetilde{\Psi}_{r}^{\infty}$ and the images of all the operators $B_{i}^{\infty}$ coincides with $\mathbb{R}^{2 n}$. Then

$$
\forall \widetilde{\Psi}(0) \in \mathbb{S}^{2 n-1} /\left\{-\widetilde{\Psi}_{r}\right\}, \quad \lim _{t \rightarrow+\infty}\left\|\widetilde{\Psi}(t)-\widetilde{\Psi}_{r}(t)\right\|=0 .
$$

Assumptions of proposition (2) are quite technical but we do not see any simplification. Controllability of the tangent linear system around each $t>0$, without any assumption regarding the asymptotic conditioning when $t \rightarrow+\infty$, does not imply asymptotic convergence of $\Psi$ towards $\Psi_{r}$. Moreover LaSalle's invariance principle becomes problematic when time dependence is not pseudo-periodic. These assumptions can be interpreted as an asymptotic controllability of the linear tangent system (21) when $t \rightarrow+\infty$.

Notice that when the analytic reference trajectory $\left(\widetilde{\Psi}_{r}, u_{r}, \omega_{r}\right)$ is pseudo-periodic, controllability of (21) automatically implies all the assumptions of proposition (2). This results from basic density and continuity arguments on the torus associated to the finite numbers of incommensurable frequencies.
The proof of theorem 6 is based on iterative applications of Barbalat's lemma: any uniformly continuous function $f:[0,+\infty) \rightarrow \mathbb{R}$ such that exits the limit of $\int_{0}^{t} f(s) d s$ when $t$ tends to $+\infty$, necessarily converges to 0 as $t \rightarrow$ $+\infty$.

PROOF. Consider proposition (1) and assume that the linear tangent system is not controllable. Take $t_{0}>0$. Then exists $v \in \mathbb{S}^{2 n-1}$ orthogonal to $\widetilde{\Psi}_{r}\left(t_{0}\right)$ such that

$$
\begin{aligned}
& B_{t=t_{0}}^{T} v=0 \\
& \forall i \in \mathbb{N}, \quad\left(\left(A-\frac{d}{d t}\right)^{i} B\right)_{t=t_{0}}^{T} v=0
\end{aligned}
$$

For any $\eta>0$, the solution $\widetilde{\Phi} \in \mathbb{S}^{2 n-1}$ of

$$
\frac{d}{d t} \widetilde{\Phi}=\left(G_{0}+u_{r} G_{1}+\omega_{r}\right) \widetilde{\Phi}
$$

with initial condition $\left(\widetilde{\Psi}_{r}\left(t_{0}\right)+\eta v\right) / \sqrt{1+\eta^{2}}$ at $t=t_{0}$ corresponds to a closed-loop trajectory. The maps

$$
t \mapsto\left\langle G_{1} \widetilde{\Phi} \mid \widetilde{\Psi}_{r}\right\rangle_{\mathbb{R}^{2 n}} \quad \text { and } \quad t \mapsto\left\langle G_{2} \widetilde{\Phi} \mid \widetilde{\Psi}_{r}\right\rangle_{\mathbb{R}^{2 n}}
$$

are analytic and all their derivatives at $t=t_{0}$ vanish. Thus they are identically zero : the closed-loop control $u$ coincides with $u_{r}$ and $\omega$ with $\omega_{r}$. Since $\left\|\widetilde{\Phi}-\widetilde{\Psi}_{r}\right\|$ is always equal to $\eta$ this is in contradiction with the assumption of proposition (1).

Let us prove proposition (2). Set

$$
\begin{aligned}
& f_{1}(t)=\left\langle G_{1} \widetilde{\Psi}(t) \mid \widetilde{\Psi}_{r}(t)\right\rangle_{\mathbb{R}^{2 n}} \\
& f_{2}(t)=\left\langle G_{2} \widetilde{\Psi}(t) \mid \widetilde{\Psi}_{r}(t)\right\rangle_{\mathbb{R}^{2 n}}
\end{aligned}
$$

Since $u_{r}, \omega_{r}$ and all their time-derivatives are bounded, $t \mapsto \Psi_{r}(t)$ and $t \mapsto \Psi(t)$ and all their time derivatives are bounded for $t \in[0, \infty)$. Thus the derivatives of $f_{1}$ and $f_{2}$ of any orders are uniformly continuous on $[0, \infty)$ and uniformly bounded. The Lyapunov function $V$ is definite positive and decreasing along the trajectory $\widetilde{\Psi}(t)$ :

$$
\frac{d}{d t} V=-a\left(f_{1}(t)\right)^{2}-b\left(f_{2}(t)\right)^{2}
$$

Take $i=1$ or 2 . By Barbalat's lemma, $f_{i}(t)$ tends to 0 as $t$ tends to $+\infty$. Since $f_{i}(t)=f_{i}(0)+\int_{0}^{t} \dot{f}_{i}$ and $f_{i}$ uniformly continuous, $\dot{f}_{i}$ tends to 0 . A simple recurrence shows that any derivative of $f_{i}$ tends to 0 . Standard computations give, for any $\nu \in \mathbb{N}$,

$$
f_{i}^{(\nu)}=\left\langle\widetilde{\Delta \Psi} \mid\left(\frac{d}{d t}-A\right)^{\nu-1} b_{i}\right\rangle_{\mathbb{R}^{2 n}}
$$


where $\widetilde{\Delta \Psi}=\widetilde{\Psi}-\widetilde{\Psi_{r}}$. Thus for any $\nu \in \mathbb{N}$

$$
\lim _{t \mapsto+\infty}\left\langle\widetilde{\Delta \Psi}(t) \mid\left(\left[\frac{d}{d t}-A\right]^{\nu-1} b_{i}\right)(t)\right\rangle_{\mathbb{R}^{2 n}}=0 .
$$

Let's suppose that $\widetilde{\Delta \Psi}$ does not converge to 0 . As $\|\widetilde{\Delta \Psi}\|(t)$ is a decreasing function of $t$ this implies that there exists a constant $\alpha>0$ such that $\|\widetilde{\Delta \Psi}\|(t)>\alpha$ for all $t>0$. Now, take the sequence $\left\{t_{k}\right\}: \overline{\Delta \Psi}\left(t_{k}\right)$ is bounded in $\mathbb{R}^{2 n}$ and so we may extract a subsequence of $\left\{t_{k}\right\}$ (which for simplicity will be still denoted as $\left.\left\{t_{k}\right\}_{k=1}^{\infty}\right)$ such that $\widetilde{\Delta \Psi}\left(t_{k}\right)$ converges to $\widetilde{\Delta \Psi}^{\infty}$ when $k \rightarrow \infty$. We have $\left\|\widetilde{\Delta \Psi}^{\infty}\right\| \geq \alpha$.

Now passing to the limit in (23) we obtain:

$$
B_{\nu}^{\infty} \widetilde{\Delta \Psi}_{\infty}=0 \quad \forall \nu \in \mathbb{N}
$$

Thus last assumption of proposition (2) implies that $\widetilde{\Delta \Psi}^{\infty}$ is co-linear to $\widetilde{\Psi}_{r}^{\infty}$, since the image of every $B_{\nu}^{\infty}$ is orthogonal to $\Psi^{\infty}$. Thus there exits $\beta \in \mathbb{R}$ such that $\widetilde{\Delta \Psi}^{\infty}=\beta \widetilde{\Psi}_{r}^{\infty}$. Set

$$
\widetilde{\Psi}^{\infty}=\lim _{k \mapsto+\infty} \widetilde{\Psi}\left(t_{k}\right) .
$$

Then $\widetilde{\Psi}^{\infty}=(1+\beta) \widetilde{\Psi}_{r}^{\infty}$. Since $\widetilde{\Psi}^{\infty}$ and $\widetilde{\Psi}_{r}^{\infty}$ belong to $\mathbb{S}^{2 n-1}$, we necessarily have $\beta=0$ or $\beta=-2$. The case $\beta=-2$ is impossible since $\left\|\widetilde{\Psi}-\widetilde{\Psi}_{r}\right\|_{t=0}<2$. Thus $\beta=0$ and we have a contradiction.

\section{Conclusion}

In this paper, we propose and analyze via theorems 1, 3 and 6 a simple Lyapunov tracking feedback (5) for any finite dimensional Schrödinger equation with a single physical control $u$. These theorems admit a straightforward extension to several controls. Such feedback design can be also extended to any infinite dimension system since it relies on the conservation of probability. However, the extension of the previous convergence analysis is not immediate since it requires the pre-compacity of the closed-loop trajectories, a property that is difficult to prove in infinite dimension.

Another natural question arises when we consider the key assumption requires by theorem 3. Assume that (1) is controllable, i.e., that the Lie algebra generated by $\imath H_{0}$ and $\imath H_{1}$ is $s u(n)$ [21]. Does there always exist $\bar{u} \in \mathbb{R}$ such that around an eigenvector of $H_{0}+\bar{u} H_{1}$, the linear tangent system is controllable. All the examples we tested validate this conjecture. Is it true for any controllable finite dimensional Schrödinger equation?

\section{References}

[1] K. Beauchard, J.-M. Coron, M. Mirrahimi, and P. Rouchon. Stabilization of a finite dimensional Schrödinger equation. Submitted to System and Control Letters, 2004.

[2] F. Blaschke. The principle of field oriention as applied to the new Transvector closed loop control system for rotating field machines. Siemens review, page 217, 1972.

[3] C. Cohen-Tannoudji, B. Diu, and F. Laloë. Mécanique Quantique, volume I\& II. Hermann, Paris, 1977.

[4] G. Espinosa and R. Ortega. An output feedback globally stable controller for induction motors. IEEE Transactions on Automatic Control, 40(1):138-143, 1995.

[5] A. Ferrante, M. Pavon, and G. Raccanelli. Control of quantum systems using model-based feedback strategies. In Proc. of the International Symposium MTNS'2002, 2002.

[6] J.P. Gauthier. Struture des Systèmes Non-Linéaires. Edition du CNRS, 1984.

[7] A. Elgart J. E. Avron. Adiabatic theorem without a gap condition. Commun. Math. Phys., 203:445-463, 1999.

[8] V. Jurdjevic and J.P. Quinn. Controllability and stability. Journal of Differential Equations, 28:381-389, 1978.

[9] T. Kato. On the adiabatic theorem of quantum mechanics. Phys. Soc. Jap., 5:435-439, 1958.

[10] T. Kato. Perturbation Theory for Linear Operators. Springer, 1966.

[11] H.K. Khalil. Nonlinear Systems. MacMillan, 1992.

[12] W. Leonhard. Control of Electrical Drives. Elsevier, 1985.

[13] Y. Maday and G. Turinici. New formulations of monotonically convergent quantum control algorithms. J. Chem. Phys, 118(18), 2003.

[14] Ph. Martin and P. Rouchon. Symmetry and field-oriented control of induction motors. Technical Report 493, Ecole des Mines de Paris, Centre Automatique et Systèmes, September 1998.

[15] André Martinez. Precise exponential estimates in adiabatic theory. J. Math. Phys., 35(B), 1994.

[16] A. Messiah. Quantum Mechanics, volume I\&II. North Holland Publ. Co., Amsterdam, 1962.

[17] M. Mirrahimi and P. Rouchon. Trajectory generation for quantum systems based on lyapounov techniques. In Proc. of IFAC Symposium NOLCOS'2004, 2004.

[18] M. Mirrahimi and P. Rouchon. Trajectory tracking for quantum systems: a lyapounov approach. In Proc. of the International Symposium MTNS'2004, 2004.

[19] M. Mirrahimi, G. Turinici, and P. Rouchon. Reference trajectory tracking for locally designed coherent quantum controls. Submitted to J. of Physical Chemistry A, 2004.

[20] W. Rabitz, H. anf Zhu. Quantum control design via adaptive tracking. J. Chem. Phys., 119(7), 2003.

[21] V. Ramakrishna, M. Salapaka, M. Dahleh, and H. Rabitz. Controllability of molecular systems. Phys. Rev. A, 51(2):960-966, 1995.

[22] J.P. Ramis and R. Schäfke. Gevrey separation of fast and slow variables. Nonlinearity, 9:353-384, 1996.

[23] S. Shi, A. Woody, and H. Rabitz. Optimal control of selective vibrational excitation in harmonic linear chain molecules. $J$. Chem. Phys., 88(11):6870-6883, 1988.

[24] L.M. Silverman and H.e. Meadows. Controllability and observability in time-variable linear systems. SIAM J. Control, 5:64-73, 1967. 
[25] M. Sugawara. General formulation of locally designed coherent control theory for quantum systems. J. Chem. Phys., 118(15):6784-6800, 2003.

[26] H.J. Sussmann and V. Jurdjevic. Controllability of nonlinear systems. J. Differential Equations, 12:95-116, 1972.

[27] G. Turinici and H. Rabitz. Wavefunction controllability in quantum systems. J. Phys. A, 36:2565-2576, 2003.

[28] P. Vettori. On the convergence of a feedback control strategy for multilevel quantum systems. In Proc. of the International Symposium MTNS'2002, 2002. 